\title{
DIFFRACTION BASED TRANSMIT-RECEIVE DELAY ELEMENT WITH ZERO TEMPERATURE COEFFICIENT
}

\author{
M. Abdelmejeed ${ }^{1 *}, J$. Kuo $^{l}$, and A. Lal ${ }^{1}$
}

${ }^{1}$ SonicMEMS Laboratory, School of Electrical and Computer Engineering, Cornell University, New York, USA

\begin{abstract}
In this paper we present a pulse-echo transmit-receive ultrasonic delay element that is stable over time and has a novel diffraction based zero temperature coefficient of delay at two temperatures. The delay element is based on a CMOS compatible aluminum nitride (AlN) piezoelectric transducer requiring no release step, further improving reliability and reducing cost of production. The silicon bulk wave propagation leads to stable operation owing to low loss within silicon and large mode volume. The delay element achieves $<6 \mathrm{ppm}$ stability and demonstrates zero temperature coefficient at $43^{\circ} \mathrm{C}$ and $72^{\circ} \mathrm{C}$.
\end{abstract}

\section{INTRODUCTION}

Previous work from our group has demonstrated on-chip ultrasonic communications [1], ultrasonic delay line memory [2], and fingerprint sensing [3] (Fig. 1) using transit and receive links consisting of $\mathrm{GHz}$ ultrasonic pulses. The scaling of CMOS circuits in size and speed favor direct integration of $\mathrm{GHz}$ ultrasonic transducers with CMOS eliminating off-chip electronics or even bonded CMOS-MEMS chips, providing a pathway for a monolithic electronic to ultrasonic interface, eliminating the need for release steps. This paper illustrates a design strategy to achieve zero temperature coefficients at any desired temperature. This could allow one to design a $37^{\circ} \mathrm{C}$, the nominal human body temperature, to measure body parameters with the delay as a reference. CMOS integrated delay-elements could be even more stable and programmable, owing to reduced parasitics.

A delay block is a circuit that shifts the input signal in time by a desired amount, and delivers a delayed output similar to the input signal. Delay elements can be used in wide range of applications including phase modulation in clocking systems as well as different digital systems. Furthermore, stable delay elements can be used as a timing reference in delay-locked-loops where the delay can be calibrated across different temperature and process variations. Precision and stability in a delay element block is one of the key specifications that directly impacts all of the aforementioned applications.

Different architectures for on-chip delay elements have been proposed in literature. The most commonly used delay method is an inverter based delay line where delay is proportional to the number of inverter buffers multiplied by the delay of a single buffer. This architecture is sensitive to process and temperature variations as well as supply voltage fluctuations. RC delay elements are also widely used in different delay based applications, however, this approach also suffers from high temperature dependence $(2500$ $\mathrm{ppm} /{ }^{0} \mathrm{C}$ ) [4]. In [5], a thyristor-based delay element is demonstrated. In this circuit, the delay is proportional to the current source as well as the threshold voltage of CMOS transistors, thus minimizing the supply dependence and hence enhancing the supply noise rejection. Minimum delay variation to power supply noise of $9.43 \%$ was reported with $314 \mathrm{PPM} /{ }^{\circ} \mathrm{C}$ temperature coefficient.

In this paper, a new ultrasonic based delay element is proposed. An ultrasonic wave packet, generated by a $75 \mathrm{umx} 75 \mathrm{um}$ AlN piezoelectric transducers, propagates through the thickness of the silicon wafer ( $\sim 25 \mathrm{um})$, where it experiences approximately total reflection at the bottom of the substrate due to the high acoustic

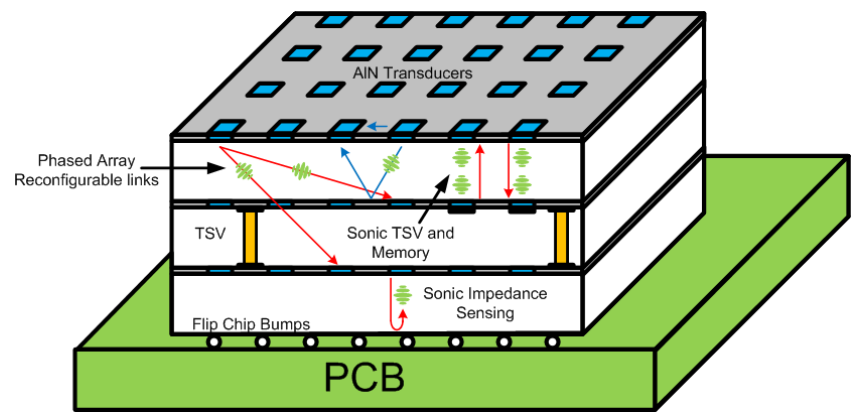

Figure 1: Applications of 3D integrated AlN transducers reconfigurable phased array communication link, sonic through silicon vias, sonic delay line memories, and integrated impedance reflectometry.

impedance mismatch between silicon and air. Due to reflection of the initial pulse and continuous diffraction of the pulse, part of the ultrasonic wave front can be received by a neighboring AlN transducer on the top of the silicon (Fig. 4). The time for the acoustic wave to be received on this transducer is the delay between the transmitted wave and the first echo. This delay can be used as an on chip stable delay reference for different timing applications.

\section{DIFFRACTION BASED TEMPERATURE COMPENSATION}

In order to analyze the diffraction patterns, consider acoustic waves induced by a piezoelectric square transducer. The pressure at an off axis observation point can be written as [8]

$p\left(\omega, x_{o}, y_{o}, z\right)=\frac{j \omega p_{o}}{2 \pi v} e^{-j\left(\frac{\omega}{v}\right)\left[z+\frac{x_{o}^{2}+y_{o}^{2}}{2 z}\right]}$
$\iint_{-\infty}^{\infty} \frac{\operatorname{rect}\left(\frac{x_{1}}{L}\right) \operatorname{rect}\left(\frac{y_{1}}{L}\right) e^{j \frac{\omega}{v z}\left(x_{o} x_{1}+y_{o} y_{1}\right)} e^{-j \frac{\omega}{v z}\left(x_{1}^{2}+y_{1}^{2}\right)}}{R} d x_{1} d y_{1}$

where $p_{o}$ is the pressure induced by the piezoelectric transducer at the transducer's surface, $\omega$ is the angular frequency of the acoustic waves, $\mathrm{v}$ is the speed of sound in silicon, $\mathrm{z}, \mathrm{x}_{0}$, and $\mathrm{y}_{\mathrm{o}}$ are the Cartesian coordinates of the observation point, and $\mathrm{L}$ is the length of the square transducer. This expression is the Fresnel diffraction expression valid for $z^{3} \gg \frac{L^{4}}{\lambda}[6]$.

The AlN pixels used have a resonant frequency of $\sim 1.3 \mathrm{GHz}$, and a lateral size of $75 \mathrm{um}$, the silicon substrate thickness is $725 \mathrm{um}$, and the speed of sound in silicon is $8433 \mathrm{~m} / \mathrm{s}$. As this thickness is much longer than the Fresnel zone length, and also satisfies the Fraunhofer approximation $\left(z \gg \frac{L^{2}}{\lambda}\right)$, equation (1) can be approximated to

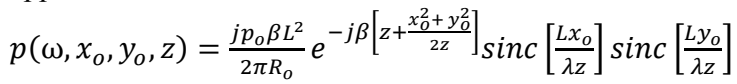

where $\beta$ is the wave number of the acoustic wave $\left(\beta=\frac{\omega}{v}\right)$. Destructive interference from the infinite point sources at the surface of the piezoelectric transducer results in nulls in the diffraction pattern along the $\mathrm{x}$-axis. This can be seen from the 
locations of the zeros in the function $\operatorname{sinc}(\mathrm{x})$, which are located at all values of $\mathrm{x}=\mathrm{n} \pi$, where $\mathrm{n}$ is an integer $(n \neq 0)$. Due to symmetry, diffraction patterns along the $\mathrm{x}$-axis and the y-axis are identical. At $y_{0}=0$, the pressure distribution along the $\mathrm{x}$-axis follows the equation

$p\left(\omega, x_{o}, y_{o}=0, z\right)=\frac{j p_{o} \beta L^{2}}{2 \pi R_{o}} e^{-j \beta\left[z+\frac{x_{0}^{2}}{2 z}\right]} \operatorname{sinc}\left[\frac{L x_{o}}{\lambda z}\right]$

where $p=0$ for $x_{o} \approx \frac{\lambda Z}{L}, \frac{2 \lambda Z}{L}, \frac{3 \lambda Z}{L}, \ldots \ldots \ldots$

From the above expressions, it can be seen that the far field diffraction angle is directly proportional to the wavelength of the propagating pulse, and inversely proportional to the size of the input piezoelectric transducer. Fig. 2 shows the plot of the diffraction pattern for 3 different wavelengths at the far field.

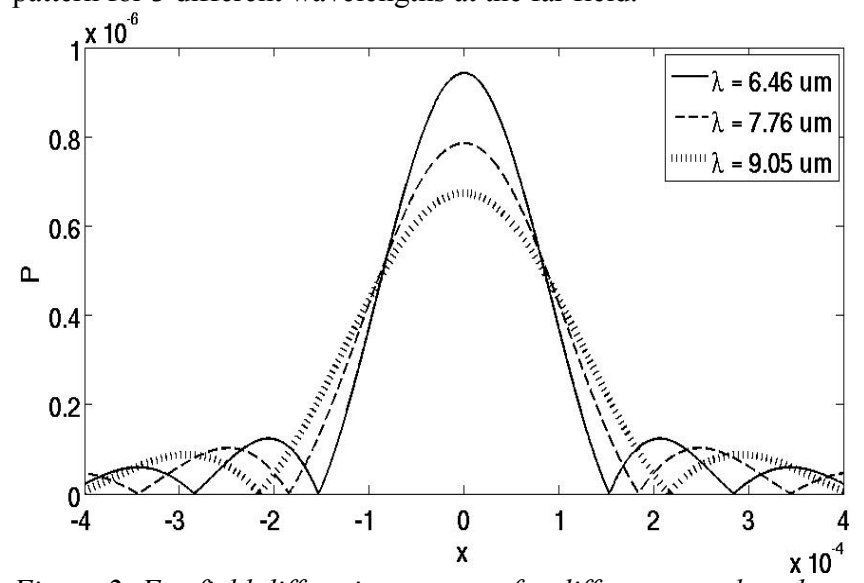

Figure 2: Far field diffraction patterns for different wavelengths at $z=1.7 \mathrm{~mm}, D=75 \mathrm{um}$.

In this setup, both the transmitting and the receiving transducers are side by side on the top of the silicon substrate. The transmitting transducer is actuated by an input pulse modulated at the resonant frequency of the transducer. The transmitted pulse is reflected at the bottom of the substrate and received by the receiving transducer. This results in the first echo for the transmitted pulse. Due to acoustic impedance mismatch at the top of the silicon substrate, most of the signal power reflects back to the substrate and takes another round trip through the silicon bulk. Throughout the rest of the paper, the signal received after one round trip will be referred to as the first echo and the signal received after two round trips will be referred to as the second echo. As an approximation, the first echo diffraction pattern will be modeled by substituting $\mathrm{Z}$ with double the silicon chip thickness $(\sim 1.45 \mathrm{~mm})$, similarly, the second echo diffraction pattern will be modelled by using a $Z$ equals to four times the chip thickness $(\sim 2.9 \mathrm{~mm})$. Fig. 3 is a schematic for the transducers on top of the silicon chip showing the dimensions used and the diffraction patterns for the first and second echoes.

The effect of temperature on the speed of sound in silicon has been discussed in detail in the literature [7]. The acoustic velocity is given by $v=\sqrt{E / \rho}, \mathrm{E}$ is the stiffness of the material and $\rho$ is its density. According to [7], for acoustic waves propagating perpendicular to the (100) Si wafer plane, the stiffness decreases approximately linearly with the temperature increase with a temperature coefficient of $-78.8 * 10^{-6} /{ }^{\circ} \mathrm{C}$. Temperature coefficient is calculated by the equation $T C=\Delta E /\left(E_{0} \Delta T\right)$, where $\Delta E$ and $\Delta T$ are the difference in stiffness and temperature respectively, and $E_{0}$ is the stiffness at room temperature.

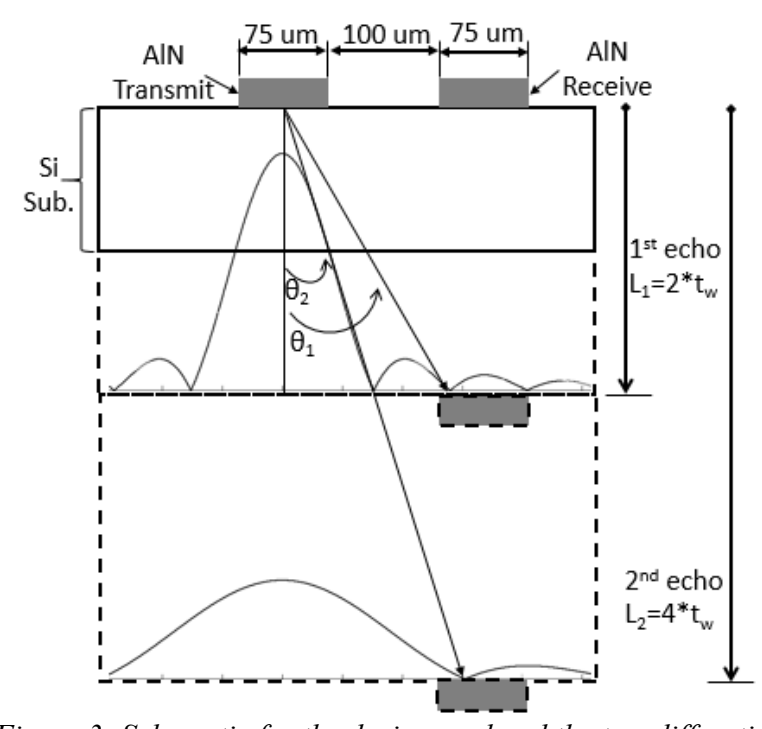

Figure 3: Schematic for the device used and the two diffraction patterns for the $1^{\text {st }}$ and $2^{\text {nd }}$ echoes.

Temperature impacts the delay of the delay cell in several ways. As temperature increases, stiffness of the delay medium decreases, and as a result, acoustic velocity is reduced. This causes delay to increase linearly with temperature. Also, thickness of silicon increases with temperature through the thermal expansion coefficient of Silicon. The total delay between the two echoes can thus be represented by the equation

$$
\begin{aligned}
& \Delta t=\delta_{2}-\delta_{1}+\left(t_{\text {rise } 2}-t_{\text {rise } 1}\right) \\
& \delta_{i}=\frac{l_{i}}{c}=\frac{1}{c_{0}(1-|\beta| \Delta T)} \frac{2 i t_{w}(1+\alpha \Delta T)}{\operatorname{Cos}\left(\theta_{i}(T)\right)} \\
& \theta_{i}=\Gamma_{i} \lambda / t_{w}=\Gamma_{\mathrm{n}} c(T) /\left(f t_{w}\right)=\frac{\Gamma_{n} c_{0}(1-|\beta| \Delta T)}{f t_{w}(1+\alpha \Delta T)}
\end{aligned}
$$

Where $\delta_{i}$ is the delay time between the initial excitation and the $\mathrm{i}^{\text {th }}$ echo due to the path length $l_{i}, t_{w}$ is the thickness of the silicon, $\mathrm{c}$ is the speed of sound, $\mathrm{T}$ is the temperature, $\mathrm{f}$ is the frequency, $\theta_{i}$ is the diffraction angle corresponding to the appropriate null, as in Fig. 3, and $\Gamma_{n}$ is an integer that corresponds to the sinc extremums.t rise $_{\text {rend }}$ and $t_{\text {rise } 2}$ are the rise times of the first and second echoes, respectively. $\beta$ is the temperature coefficient by which the speed of sound decreases in silicon $\left(\sim-50 \mathrm{ppm} /{ }^{\circ} \mathrm{C}\right) . \alpha$ is the thermal expansion coefficient of Silicon $\left(\sim 2.6 \mathrm{ppm} /{ }^{\circ} \mathrm{C}\right)$. These values indicate that the delay is always a rising function of temperature.

However, as shown in equation (4), additional delay is present due to the circuit effect on measuring the rise time. In Fig. 2, as the wavelength decreases, angle of diffraction decreases. Consequently, the amount of received power changes, resulting in changing the rise time of the received echoes which in turn changes the delay. As an example, in Fig. 3, in the case of the first echo, as temperature increases, the third peak of the diffraction pattern moves away from the receiver transducer range. Consequently, the amount of signal power received at higher temperature is less than the amount received at room temperature. Conversely, for the second echo, as temperature increases, the first peak in the diffraction pattern moves towards the range of the receiver transducer. As a result, the amount of signal power received gets higher. As will be shown later, the rise time of the echo pulse is inversely proportional to the received signal amplitude (total received power). So, $t_{\text {rise }}$ increases and $t_{\text {rise } 2}$ decreases causing the total delay in equation (4) to decrease. After a certain threshold the behavior will be reversed and the received signal amplitude increases with temperature at the first echo and 
decreases with temperature at the second echo causing the delay between the first and the second echoes to increase. Fig. 4 shows how the received signal amplitude changes with temperature for the first and the second echoes.

An envelope detector is used to down convert the signal from the resonant frequency of the transducer to baseband. We can model the electronic circuit as a rectifier. The signal is rectified using a half wave rectifier Zener diode, then a capacitor is charged by the rectified wave to track the envelope of the signal. The change of rise time is related to the change in the signal amplitude (received signal power) by the capacitor charging equation

$$
\begin{gathered}
t_{\text {rise }}=R_{\text {eff }} C \ln \left(1+\frac{V_{T}}{V_{\text {peak }}}\right) \\
\Delta t_{\text {rise }}=t_{\text {rise } 2}-t_{\text {rise } 1}=R_{\text {eff }} C \ln \left(\frac{V_{\text {Peak } 1}\left(V_{\text {Peak } 2}+V_{T}\right)}{V_{\text {Peak } 2}\left(V_{\text {Peak } 1}+V_{T}\right)}\right)
\end{gathered}
$$

$t_{\text {rise }}$ is the pulse rise time in sec, $R_{\text {eff }}$ is the effective resistor charging the capacitor in ohms, $C$ is the capacitance of the capacitor in Farad, $\mathrm{V}_{\mathrm{T}}$ is the switching threshold voltage, and $\mathrm{V}_{\text {peak }}$ is the input signal amplitude. $V_{\text {Peak1 }}$ and $V_{\text {Peak2 }}$ are normally very close to each other in value, hence, equation (5) can be approximated by the Taylor expansion of the first term giving

$$
\begin{aligned}
\Delta t_{\text {rise }} & \approx R_{\text {eff }} C\left(\frac{V_{\text {Peak } 1}\left(V_{\text {Peak } 2}+V_{T}\right)}{V_{\text {Peak } 2}\left(V_{\text {Peak } 1}+V_{T}\right)}-1\right) \\
\Delta t_{\text {rise }} & \approx \frac{-R_{\text {eff }} C V_{T} \Delta V_{\text {Peak }}}{V_{\text {Peak }}\left(V_{\text {Peak }}+V_{T}\right)}
\end{aligned}
$$

From (6), as the received power changes $\left(\mathrm{V}_{\text {Peak }}\right)$, the rise time also changes. At small variation in peak voltage, $\Delta t_{\text {rise }}$ changes linearly with $\Delta \mathrm{V}_{\text {peak }}$ and the coefficient of variation is proportional to the envelope detector circuit parameters $\left(\mathrm{R}_{\mathrm{eff}}\right.$ and $\left.\mathrm{C}\right)$. This second term has a larger component of delay measurement, and makes the delay sensitive to a resistor and capacitance. Hence, if on-chip resistors and capacitors are used, the delay could be susceptible to on-chip process variations and components aging.

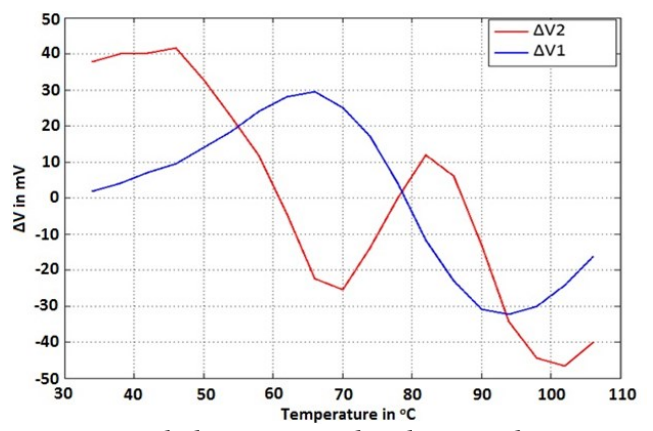

Figure4: Measured change in peak voltage with temperature for the two pulses

\section{EXPERIMENT SETUP}

The experiment setup (Fig. 5) comprises an RF signal generator, a pulse generator, an RF switch, the transducers device, a thermocouple to measure the temperature, an oven, an envelope detector, and a frequency counter with high precision to accurately measure the delay. The RF switch is controlled by the pulse generator to create pulses modulated by the high frequency signal generated by the RF signal generator. The pulse width should be less than the time of flight of acoustic waves in silicon so that echoes do not overlap. In our experiment, time of flight for a round trip in silicon is $\sim 175 \mathrm{~ns}$, so, the pulse width was chosen to be $80 \mathrm{~ns}$. The pulse is then used to actuate the transmit AlN transducer. The wave generated by the piezoelectric transducer of width 75 um propagates through the thickness of a silicon wafer as illustrated in Fig. 4. The envelope detector is then used to down convert the received signal and the frequency counter is used to measure the delay between the first 2 echoes.

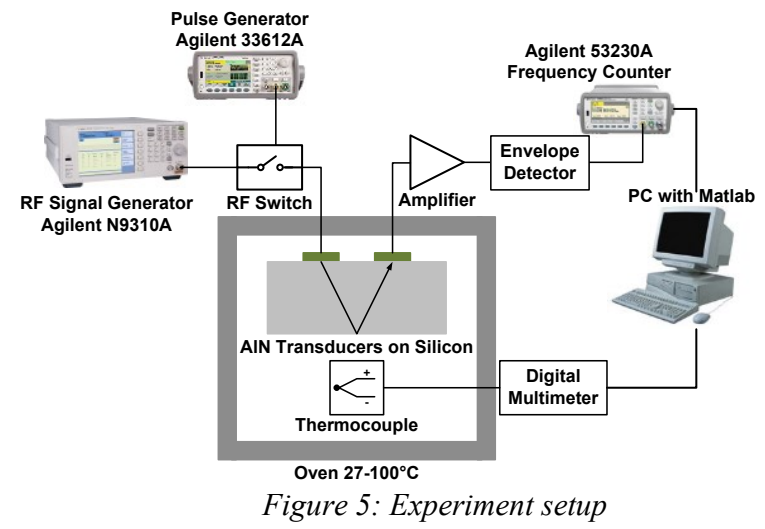

\section{MEASUREMENT RESULTS}

Fig. 6 shows the measured signals at the output of the amplifier and the output of the envelope detector. The first pulse is due to electrical coupling of the transmit signal to the receive transducer. This is followed by several acoustic echoes. In this paper, the reference delay of interest is the delay between the first and the second echo. However, the same analysis applies to the delay between all consecutive echoes. At room temperature, the delay between each two consecutive pulses is $\sim 175 \mathrm{~ns}$. The input frequency to the envelope detector is $1.31 \mathrm{GHz}$ which is the resonance frequency of the piezoelectric transducer.

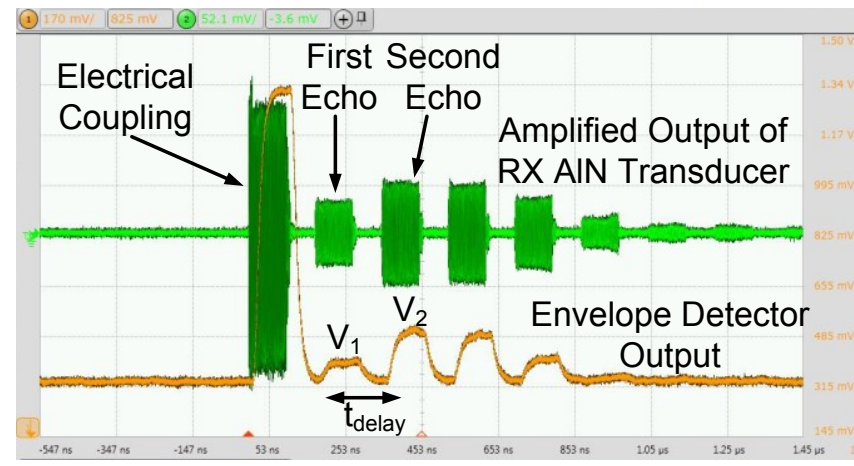

Figure 6: Experiment waveforms showing the delay of interest.

The Allan deviation curve is calculated for the delay of interest as a metric of stability. The Allan deviation is the square root of the Allan variance, which is half of the average of the squares of the differences between successive delay readings over a certain time period. The Allan deviation allows different types of noise to be distinguished based on the Allan curve slope when plotted on loglog plot. Fig. 7 shows the Allan deviation curve for the delay between the first and the second echoes. The curve starts with a slope of $-1 / 2$ due to white noise, then slope starts to decrease until it flattens at the flicker noise floor, where more averaging doesn't help in noise reduction. Unlike the conventional Allan deviation curves, the curve doesn't start with a -1 slope which means that the system doesn't suffer from quantization noise. A minimum standard deviation of $0.87 \mathrm{ps}$ is achieved which corresponds to a delay error of 5 ppm. 


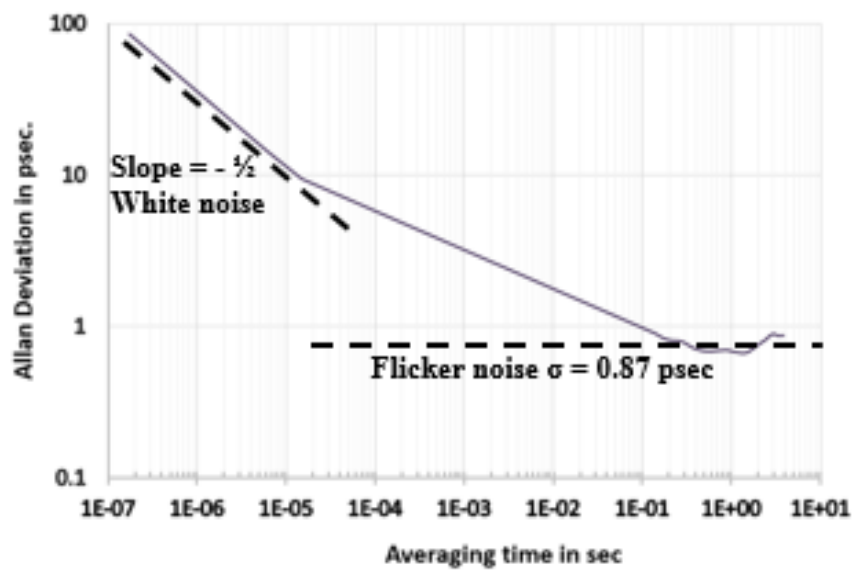

Figure 7: Allan deviation of $t_{\text {delay. }}$ Averaging delays over 4 seconds results in an Allan deviation of $0.87 \mathrm{ps}$

Fig 8. shows the measured delay across temperature. The effect of the diffraction pattern change discussed earlier can be clearly seen. The temperature effect on $\mathrm{t}_{\text {delay }}$ in $\mathrm{PPM} /{ }^{\circ} \mathrm{C}$ is in Fig. 9. Around $43^{\circ} \mathrm{C}$ and $72^{\circ} \mathrm{C}$, the temperature coefficient is 0 . These values can be changed through the experiment setup parameters like the receiver position, the actuation frequency, and the effective resistance and the output capacitance of the envelope detector, allowing one to program to $37^{\circ} \mathrm{C}$, body temperature, for human body sensing modalities.

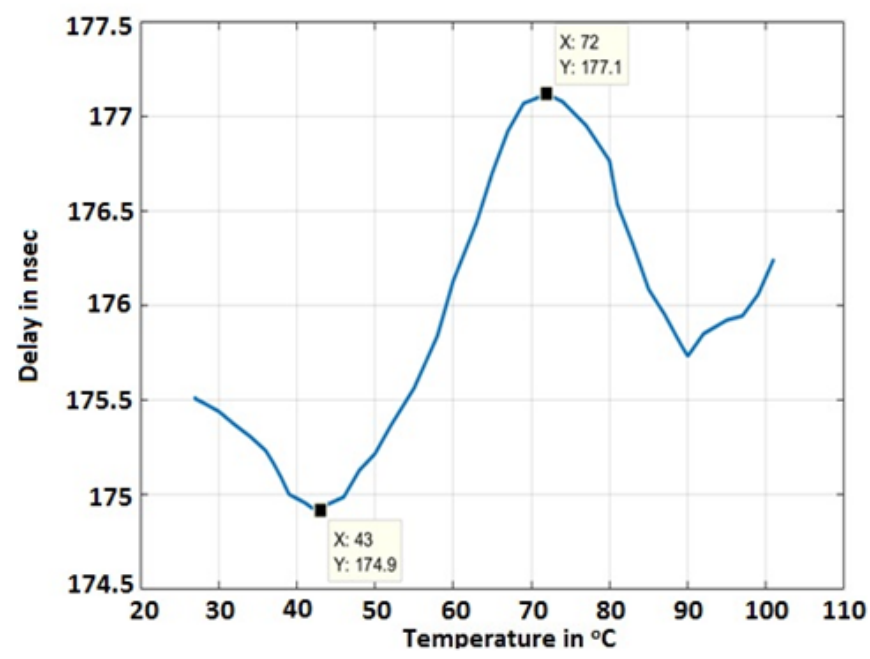

Figure 8: Measured delay between the first and the second echoes across temperature

\section{CONCLUSION}

In this paper, a CMOS compatible delay element based on ultrasound pulse packet propagation through the silicon bulk is introduced. The delay element showed high long term stability with an Allan deviation of $0.87 \mathrm{ps}(<6 \mathrm{ppm})$. Also, due to the diffraction angle dependence on temperature, a zero temperature coefficient is achieved at $43^{\circ} \mathrm{C}$ and $72^{\circ} \mathrm{C}$. The transmit-receive geometries can be designed with different spacing, higher number of electrodes for differential delay measurement and differential receive electronic characteristics. Further design is needed for delay-locked loops to investigate the stability of frequency sources.

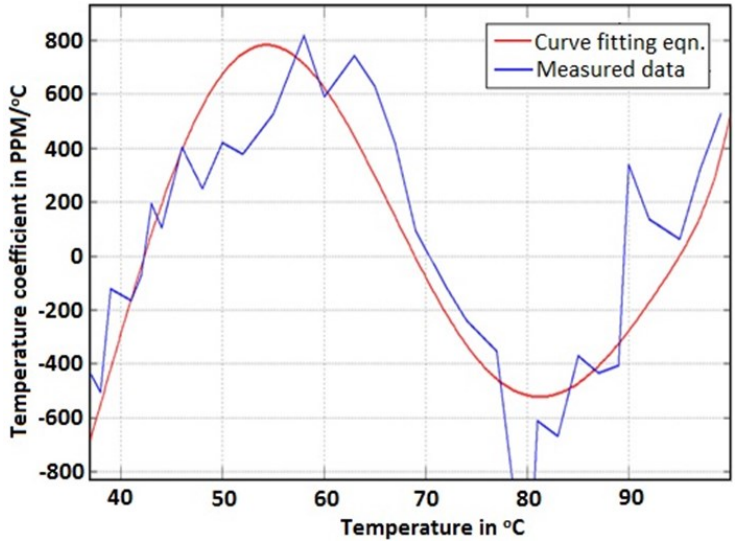

Figure 9: Temperature coefficient in $P P M /{ }^{\circ} \mathrm{C}$ across temperature showing measured data and a fitted curve.

\section{ACKNOWLEDGMENT}

The authors would like to acknowledge the IARPA Trusted Integrated Chips (TIC) program for funding and Cornell Nanofabrication Facility (CNF) for use of facilities and equipment (ECCS-1542081).

\section{REFERENCES}

[1] J. Hoople, J. Kuo, S. Ardanuc, and A. Lal, "Chip-scale reconfigurable phased-array sonic communication," in Ultrasonics Symposium (IUS), 2014 IEEE International, 2014, pp. 479-482.

[2] J. Kuo, J. Hoople, S. Ardanuc, and A. Lal, "Towards ultrasonic through-silicon vias (UTSV)," in Ultrasonics Symposium (IUS), 2014 IEEE International, 2014, pp. 483-486.

[3] J. Hoople, J. Kuo, M. Abdel-moneum, and A. Lal, "Chipscale $\mathrm{GHz}$ ultrasonic channels for fingerprint scanning," in 2015 IEEE International Ultrasonics Symposium (IUS), 21-24 Oct. 2015

[4] N. R. Mahapatra, S. V. Garimella and A. Tareen, "An empirical and analytical comparison of delay elements and a new delay element design," VLSI, 2000. Proceedings. IEEE Computer Society Workshop on, Orlando, FL, 2000, pp. 81-86.

[5] J. Zhang, S. R. Cooper, A. R. LaPietra, M. W. Mattern, R. M. Guidash and E. G. Friedman, "A low power thyristor-based CMOS programmable delay element," Circuits and Systems, 2004. ISCAS '04. Proceedings of the 2004 International Symposium on, 2004, pp. I-769-72 Vol.1.

[6] J. W. Goodman, Introduction to Fourier Optics. Englewood, Colorado: Roberts \& Co., 2005.

[7] C. Bourgeois, E. Steinsland, N. Blanc and N. F. de Rooij, "Design of resonators for the determination of the temperature coefficients of elastic constants of monocrystalline silicon," Frequency Control Symposium, 1997., Proceedings of the 1997 IEEE International, Orlando, FL, 1997, pp. 791-799.

[8] R. S. C. Cobbold, "Field Profile Analysis," in Foundations of Biomedical Ultrasound. New York: Oxford, 2007, ch. 3, pp. 185-189.

\section{CONTACT}

*M. Abdelmejeed, tel: +1-607-379-9712; moa35@cornell.edu 